\title{
La grande course des universités
}

Christine Musselin, Les Presses de Sciences Po, 2017, 304 p.

\section{Daniel Coste}

\section{(2) OpenEdition}

\section{Journals}

Édition électronique

URL : https://journals.openedition.org/ries/6110

DOI : $10.4000 /$ ries. 6110

ISSN : 2261-4265

Éditeur

France Education international

Édition imprimée

Date de publication : 30 avril 2018

Pagination : 33-34

ISBN : 978-2-85420-618-0

ISSN : $1254-4590$

\section{Référence électronique}

Daniel Coste, "La grande course des universités », Revue internationale d'éducation de Sèvres [En ligne],

77 | avril 2018, mis en ligne le 30 avril 2018, consulté le 25 juin 2021. URL : http://

journals.openedition.org/ries/6110; DOI : https://doi.org/10.4000/ries.6110

Ce document a été généré automatiquement le 25 juin 2021.

(c) Tous droits réservés 


\title{
La grande course des universités
}

\author{
Christine Musselin, Les Presses de Sciences Po, 2017, 304 p.
}

Daniel Coste

1 L'époque où les doyens de faculté hantaient les couloirs des ministères et y obtenaient crédits ou mesures nouvelles pour leur secteur de responsabilité est désormais bien révolue. C'est un bouleversement du paysage des universités françaises que Christine Musselin, directrice de recherche au CNRS, directrice scientifique de Sciences Po et chercheuse au Centre de sociologie des organisations (CSO) propose à ses lecteurs. L'ouvrage est très riche de références et de mention des sources, mais reste de lecture très aisée en dépit de sa technicité et de la complexité des dispositifs et des évolutions dont il rend compte. Bouleversement du paysage en effet, d'autant plus marqué que les effets d'une certaine mondialisation et d'une compétition accrue affectent une situation universitaire française longtemps très distincte de celles de ses homologues étrangères (et que rappelle un très utile chapitre à caractère historique). Dans l'introduction à ses analyses et en illustration du titre du livre, Christine Musselin compare ce bouleversement à une course cycliste. Longtemps les universités ont avancé plus ou moins en peloton et à allure moyenne sur un terrain relativement plat. Aujourd'hui, elles se trouvent engagées dans une étape de montagne où l'on s'affronte pour la première place, où le peloton a éclaté et où certaines ont irrémédiablement décroché.

2 Le cadre général dans lequel l'auteure s'inscrit est celui des rapports triangulaires entre instance de tutelle, université et profession universitaire. Sans surprise, Christine Musselin souligne l'incidence des dispositifs nationaux et internationaux d'évaluation dans les transformations du paysage universitaire. Les contrats quadriennaux (aujourd'hui quinquennaux), initiés par Lionel Jospin dans un gouvernement de Michel Rocard, ont fait des universités et de leurs instances de gestion des partenaires majeurs (au détriment des facultés) et ont induit, sans même que cette réforme ait vraiment abouti, une plus grande autonomie des universités, une logique de projet, des politiques d'établissements appelés à remettre en cause les cloisonnements disciplinaires, au profit d'une affirmation différentielle des universités les unes par rapport aux autres. 
Surtout, les évaluations de l'ANR (Agence nationale de la recherche), les expertises de l'AERES (Agence d'évaluation de la recherche et de l'enseignement supérieur), les appels à projets nationaux, la labellisation, la sélection de "laboratoires d'excellence " (Labex) ont progressivement installé, depuis le début des années 2000, une culture de la mise en compétition. C'est un abandon des discours uniformisants et une rupture nette avec le politiquement correct antérieur, qui affirmait l'égalité des territoires et des trajectoires. Dans cette dynamique nouvelle, non seulement les différences se creusent, y compris en termes de moyens, entre les primés et ceux qui ne le sont pas, mais on relève aussi que l'évaluation classante des universités et des équipes rejaillit sur l'appréciation des chercheurs individuels et sur leurs modalités de travail.

Les effets de ces orientations sont notamment perceptibles en sciences humaines: encouragement aux travaux collectifs, à l'interdisciplinarité, aux projets regroupant plusieurs établissements. On voit évoluer les modes de publication, ne serait-ce qu'à la marge: valorisation des articles (dans des revues indexées à reconnaissance internationale) et pas seulement des livres, collaboration de plusieurs auteurs pour un même article, formalisation et standardisation croissantes des normes et formats de publication, etc.

5 Pour les multiples dispositifs mis en œuvre, les critères d'évaluation et le choix des évaluateurs donnent évidemment lieu à interrogations et à débats. Si le principe de l'évaluation par les pairs est maintenu, l'examen des grands projets s'internationalise (ce qui, soit dit au passage, n'est pas sans incidence sur les dimensions linguistiques du processus d'expertise et sur la rédaction même des projets soumis en réponse à appels). Car l'internationalisation des classements (le fameux classement de Shanghai des universités mondiales) pèse fortement sur les stratégies de pilotage national et sur les choix des universités qui sont (encore) dans la course. L'organisation de la compétition entre universités se double d'une forme d'injonction au regroupement local et régional entre établissements (grandes écoles, organismes de recherche, universités), qui vise à faire masse et à peser plus dans les classements par regroupement des forces. Non sans quelques grincements.

6 Les transformations en cours sont profondes et s'accélèrent, mais il est trop tôt pour mesurer leur impact. Comme le résume la quatrième de couverture de cet ouvrage aux analyses aussi lucides que nécessaires :

De nouvelles structures sont ainsi créées à marche forcée, sans qu'il soit possible de savoir si ces changements majeurs atteindront leurs objectifs et assureront un avenir radieux à l'enseignement supérieur français.

\section{AUTEUR}

\section{DANIEL COSTE}

Daniel Coste, ancien directeur de recherches à l'Université Paris 3 et ancien responsable à l'École normale supérieure de Lyon de l'équipe de recherche « Plurilinguisme et apprentissages », a dirigé le Centre de recherche et d'étude pour la diffusion du français (1992-2003) et enseigné la 
linguistique appliquée à l'Université de Genève (1988-1992). Auteur ou co-auteur d'ouvrages, chapitres et articles en didactique des langues et en histoire de l'enseignement des langues, associé aux travaux de l'Unité des politiques linguistiques du Conseil de l'Europe, il a fait partie du groupe d'auteurs du Cadre européen commun de référence pour les langues. Courriel :

costedaniel@sfr.fr 